
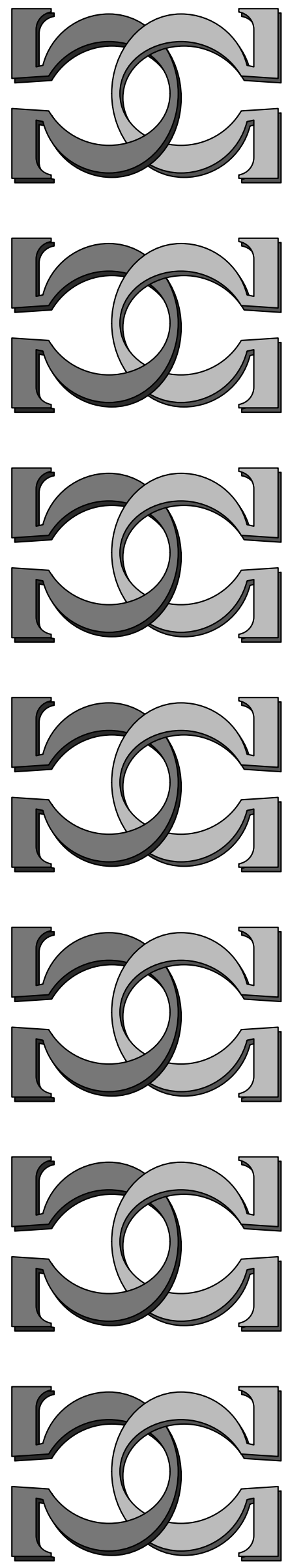

\section{CDMTCS}

Research

Report

Series

\section{Degree-Theoretic Aspects of Computably Enumerable Reals}

Cristian S. Calude Richard Coles Peter H. Hertling Bakhadyr Khoussainov

Department of Computer Science University of Auckland

CDMTCS-090

September 1998

Centre for Discrete Mathematics and Theoretical Computer Science 


\title{
Degree-Theoretic Aspects of Computably Enumerable Reals
}

\author{
Cristian S. Calude, Richard Coles, Peter H. Hertling \\ and Bakhadyr Khoussainov
}

September 21, 1998

\begin{abstract}
A real $\alpha$ is computable if its left cut, $L(\alpha)$, is computable. If $\left(q_{i}\right)_{i}$ is a computable sequence of rationals computably converging to $\alpha$, then $\left\{q_{i}\right\}$, the corresponding set, is always computable. A computably enumerable (c.e.) real $\alpha$ is a real which is the limit of an increasing computable sequence of rationals, and has a left cut which is c.e. We study the Turing degrees of representations of c.e. reals, that is the degrees of increasing computable sequences converging to $\alpha$. For example, every representation $A$ of $\alpha$ is Turing reducible to $L(\alpha)$. Every noncomputable c.e. real has both a computable and noncomputable representation. In fact, the representations of noncomputable c.e. reals are dense in the c.e. Turing degrees, and yet not every c.e. Turing degree below $\operatorname{deg}_{T} L(\alpha)$ necessarily contains a representation of $\alpha$.
\end{abstract}

\section{Introduction}

Computability theory essentially studies the relative computability of sets of natural numbers. Since Gödel introduced a method for coding structures using natural numbers $\Gamma$ computability has been applied to many areas of mathematics $\Gamma$ for example $\Gamma$ to the theory of linear orders $\Gamma$ to group theory and to real analysis. In this paper we will consider an application of computability theory to the real numbers.

The real numbers $\mathbb{R}$ may be defined in several different ways. In classical analysis $\Gamma$ the reals are those entities which are the limit of a Cauchy sequence. In mathematical logic the real numbers are defined as Dedekind cuts of sets. Robinson [12] considered effective versions of Cauchy sequences and Dedekind cuts. In this paper the effective version of both Dedekind cuts and Cauchy sequences play a role. 
We first consider effective converging sequences of rationals. A computable real number will be one that can be expressed as the limit of a computable sequence of rationals that converges computably. Alternatively $\Gamma$ and equivalently $\Gamma$ a computable real can be defined as a real with a computable left (or right) Dedekind cut. All relevant notions will be made formal in later sections.

It follows that every rational is a computable real and further $\Gamma$ that many well-known irrationals are computable $\Gamma$ for example $\pi$ and $e$. Furthermore $\Gamma$ the set of all computable reals is a real-algebraically closed field.

Specker [15] gave the first example that computable sequences of rationals may converge in a noneffective way. He coded the Halting Problem into a computable sequence of rationals and then showed that if this sequence converged computably then there would be an algorithm for computing the Halting Problem.

For a similar reason $\Gamma$ it is not possible to decide whether a computable sequence of rationals that converges computably $\Gamma$ converges to 0 say. Hence the equality between two computable reals is also undecidable. (See Rice [11] or Calude [2] for example.)

We now consider effective Dedekind cuts. A natural set associated with a real $\alpha$ is $L(\alpha)=\{q \in \mathbb{Q} \mid q<\alpha\}$; it corresponds to the left Dedekind cut of the real $\alpha$. Soare [13] studied computability theoretic properties of Dedekind cuts. With a subset $A$ of natural numbers we associate the real $\alpha=0 . A(0) A(1) A(2) \ldots$ where $A(i)=1$ if $i \in A$ and $A(i)=0$ if $i \notin A$. We also write $\alpha=0 \cdot \chi_{A}$. By interpreting this as a binary string we observe that we are dealing with reals in the interval $[0,1]{ }^{1}$

Soare showed $\Gamma$ for example $\Gamma$ that $A \leqslant t t L(\alpha)$ but $L(\alpha)$ is not necessarily truth-table reducible to $A$, although $L(\alpha) \leqslant_{T} A$. Furthermore $\Gamma L(\alpha)$ is always a semirecursive set. (See Jockusch [6] for much more on semirecursive sets.)

It is not difficult to see that $A$ is a computable set if and only if $L(\alpha)$ is a computable set. As Soare points out $\Gamma$ if we replace computable by computably enumerable $\Gamma$ then this equivalence no longer holds. If $A$ is a computably enumerable set then $L(\alpha)$ is also a computably enumerable set. However the converse fails. In this paper we will define the property of a set being strongly $\omega$-c.e. and see that if $L(\alpha)$ is computably enumerable then $A$ is a strongly $\omega$-c.e. set. This observation is made in Calude et al [4ГTheorem 4.1].

In a lot of work in computable analysis $\Gamma$ emphasis has been put on computability and the question whether certain objects (real numbers $\Gamma$ functions $\Gamma$

\footnotetext{
${ }^{1}$ Our choice of which real to associate with a set of natural numbers differs slightly from that in Soare [13]. With a subset $A$ of natural numbers Soare associated a real number in the interval [0,2], namely $\Phi(A)=\Sigma_{n \in A} 2^{-n}$, and $\Phi(\emptyset)=0$. However from the point of view of Turing reducibility there is no difference because if $\alpha=0 . \chi_{A}$ then $\Phi(A)=2 \alpha$, hence $L(\alpha) \equiv_{t t} L(\Phi(A))$ and $A \equiv_{T} L(\alpha) \equiv_{T} L(\Phi(A))$.
} 
etc.) are computable or not. Less is known about noncomputable real numbers. See Pour-El and Richards [10] or Weihrauch [17] for a development of computable analysis. We also cite Martin-Löf [9] and Bridges and Richman [1].

A computably enumerable real is defined to be the limit of an increasing computable sequence of rationals. In this paper we focus on the Turing degrees of increasing computable sequences of rationals that converge to some real $\alpha$. The layout of the paper is the following.

We begin with some notation in Section 2. In Section 3 Cfirst we consider Turing degrees of computable sequences of rationals which converge computably. The only degree we get this way is $\boldsymbol{O}$. Then we consider Turing degrees of arbitrary computable sequences of rationals. It turns out that we can fix an arbitrary real number which can be obtained as the limit of such a sequence and restrict ourselves to sequences with this limit $\Gamma$ and still get all c.e. degrees. Then we introduce computably enumerable reals.

In Section 4 we take a closer look at the Turing degrees of increasing computable sequences converging to c.e. reals. It turns out that the set of degrees obtained by looking at increasing computable sequences of rationals converging to a fixed c.e. real has a lot to do with splitting. Any such degree is below $L(\alpha)$. We show that for every noncomputable c.e. real $\alpha$, there are infinitely many c.e. Turing degrees that contain sets of rationals which are increasing computable sequences converging to $\alpha$.

The construction of increasing computable sequences converging to a real $\alpha$ is a dynamic process $\Gamma$ and so many computability strategies that depend on waiting for some situation to occur in a construction $\Gamma$ for example waiting for a partial computable function to halt on a particular argument $\Gamma$ or waiting for some number to be enumerated into a set $\Gamma$ are in conflict with such a construction. Because of this Tin Section 5 we are able to show that there is a computably enumerable real $\alpha$ and a computably enumerable Turing degree below $L(\alpha)$ that does not contain an increasing computable sequence that converges to $\alpha$. We conclude the paper with Section 6 by stating some open questions suggested by the above results.

For more background on computability theory see Soare [14] for example.

\section{Preliminaries}

When dealing with computability we think in terms of computations on natural numbers. In this paper we are concerned with constructing sequences of rationals having certain computability theoretic properties $\Gamma$ and so we fix a standard computable bijection $\theta: \mathbb{Q} \mapsto \mathbb{N}$. When we work with sets of rationals we identify them with the subsets of $\mathbb{N}$ that are their images under 
$\theta$, and similarly we move from sets of natural numbers to sets of rationals via $\theta^{-1}$. If $A \subseteq \mathbb{Q}$ then we write $\theta(A)$ for the set $\{\theta(q) \mid q \in A\}$, and similarly for $B \subseteq \mathbb{N}$ and $\theta^{-1}(B)$.

For a set $A$ we write $|A|$ to denote the cardinality of $A$. We let $A \backslash B$ denote $\{x \mid x \in A \& x \notin B\}$. For a set $A$, we define $A(i)=1$ if $i \in A$ and $A(i)=0$ if $i \notin A$. We write $A \uparrow_{x}$ for the set $\{n \mid n \in A \& n<x\}$. If $A$ is a set of natural numbers then we write $\alpha=0 . \chi_{A}$ to denote the real $\alpha=0 . A(0) A(1) A(2) \ldots \Gamma$

Let $\Phi_{0}, \Phi_{1}, \ldots$ be a standard listing of all Turing functionals. We write $\Phi_{e}(A)$ to denote the $e$-th functional acting on oracle $A$. We often write $\Phi_{e}$ in place of $\Phi_{e}(\emptyset)$. If $\Phi(A)(x)$ is a halting computation then we write $\Phi(A)(x) \downarrow$, and similarly $\Gamma$ if $\Phi(A)(x)$ is not a halting computation then we write $\Phi(A)(x) \uparrow$. We append $[s]$ to parameters to denote their value after $s$ steps $\Gamma$ for example $\Phi(A)(x) \uparrow[s]$ means that the computation $\Phi(A)(x)$ has not halted after $s$ steps.

Let $W_{e}$ denote the $e$-th computably enumerable set $\Gamma$ that is $W_{e}=\operatorname{dom} \Phi_{e}$. We let $K$ denote the set $\left\{x \mid \Phi_{x}(x) \downarrow\right\}$. We relativise $K$ to subsets $A \subseteq \mathbb{N}$, writing $K^{A}$ for the set $\{x \mid \Phi(A)(x) \downarrow\}$.

If $A=\Phi(B)$ for some Turing functional $\Phi$ then we say that $A$ is Turing reducible to $B$ and write $A \leqslant_{T} B$. Further $\Gamma$ we let $A \equiv_{T} B$ denote $A \leqslant_{T} B$ and $B \leqslant_{T} A$. We form equivalence classes of subsets of $\mathbb{N}$ via the equivalence relation $\equiv_{T}$ and write $\boldsymbol{a}, \boldsymbol{b}$, and so on to denote the equivalence classes. We define $\boldsymbol{a} \leqslant \boldsymbol{b}$ if there is some $A \in \boldsymbol{a}$ and $B \in \boldsymbol{b}$ such that $A \leqslant_{T} B$. The Turing degrees then form a partial order with respect to the ordering above which we denote by $\mathcal{D}(\leqslant)$.

The Turing jump operator is defined on subsets of $\mathbb{N}$ as $A^{\prime}=K^{A}$. Hence $K \equiv_{T} \emptyset^{\prime}$. We say a set $A$ is low if $A^{\prime} \equiv_{T} K$. Recall that a set $A$ is $\Delta_{2}^{0}$ if and only if $A \leqslant_{T} K$.

Define $A \oplus B=\{2 n \mid n \in A\} \cup\{2 n+1 \mid n \in B\}$. It is not difficult to see that $\operatorname{deg}_{T}(A \oplus B)$ is the least upper bound of $\operatorname{deg}_{T}(A)$ and $\operatorname{deg}_{T}(B)$, and so $\mathcal{D}(\leqslant)$ forms an upper semi-lattice. Whenever we simply write degree it is understood to be Turing degree.

We define the degree of a real $\alpha, \operatorname{deg}_{T}(\alpha)$, to be the degree of $A \Gamma$ where $0 . \chi_{A}$ is the fractional part of $\alpha$. Note that there is either a unique such set $A$ or there are two Tone finite and one cofinite.

With a finite set $X=\left\{x_{1}, x_{2}, \ldots x_{k}\right\}$ we can associate the canonical index $y=2^{x_{1}}+2^{x_{2}}+\ldots+2^{x_{k}}$. Let $D_{y}$ denote the finite set with canonical index $y$ and $D_{0}$ denotes $\emptyset$. A computable approximation to a $\Delta_{2}^{0}$ set $A$ is a sequence $\left(D_{f(i)}\right)_{i}$ of sets $D_{f(i)}$ for $i \in \mathbb{N}$ for some computable function $f$ such that $A(x)=\lim _{i} D_{f(i)}(x)$.

We denote sequences of rationals by $\left(q_{i}\right)_{i}$ and by increasing sequence we shall mean strictly increasing. For $q \in \mathbb{Q}$ we define $q(x)=i$ if the $x$ th bit of the binary representation containing infinitely many ones of the fractional 
part of $q$ is $i$.

\section{Computable sequences of rationals}

We consider the Turing degrees of two classes of computable sequences of rationals and introduce computably enumerable reals.

Definition 1. A sequence $\left(q_{i}\right)_{i}$ of rationals is called computable if the total function $g: \mathbb{N} \mapsto \mathbb{N}$ defined by $\theta^{-1} \circ g(i)=q_{i}$ for all $i$ is computable.

So a sequence is computable if we can (uniformly) effectively decide the $i$ th member. However $\Gamma$ we may be unable to decide the rationals that do not occur in the sequence. If $\left(q_{i}\right)_{i}$ is a sequence of rationals $\Gamma$ we denote the set $\left\{q \in \mathbb{Q} \mid \exists i \in \mathbb{N}\left(q=q_{i}\right)\right\}$ by $\left\{q_{i}\right\}$. For computable sequences of rationals it is obvious that $\left\{q_{i}\right\}$ is a computably enumerable set. We will be interested in the Turing degrees of such sets.

First we look at sequences which define the "simplest" kind of real number $\Gamma$ in terms of computability.

Definition 2. A sequence $\left(r_{i}\right)_{i}$ of reals converges computably if there is a total computable function $g: \mathbb{N} \mapsto \mathbb{N}$ such that for each $n \Gamma\left|r_{i}-\alpha\right| \leqslant 2^{-n}$ for all $i \geqslant g(n)$. We call $g$ a modulus of convergence function for $\left(r_{i}\right)_{i}$.

Definition 3. A real $\alpha$ is computable if there is a computable sequence of rationals that converges computably to $\alpha$.

As noted in the introduction $\Gamma$ we could equivalently define computable reals to be those reals $\alpha$ such that $L(\alpha)$ is a computable set.

In this paper we do not concern ourselves with convergent computable sequences having a noncomputable modulus of convergence. The interested reader should consult Calude and Hertling [3] for a study.

Theorem 4. If a sequence $\left(q_{i}\right)_{i}$ of rationals is computable and converges computably, then the set $\left\{q_{i}\right\}$ is computable.

Proof. Let $\left(q_{i}\right)_{i}$ be a computable sequence of rationals converging computably to $\alpha$. Then there is a total computable function $g$ such that for each $n$, $\left|q_{i}-\alpha\right| \leqslant 2^{-n}$ for all $i \geqslant g(n)$. We give a procedure for deciding if $p \in\left\{q_{i}\right\}$ for an arbitrary rational $p$. We distinguish three cases.

(1) $\alpha$ is irrational.

To decide $p \in\left\{q_{i}\right\}$ perform the following procedure:

Enumerate intervals $\left(q_{k}-2^{-n+1}, q_{k}+2^{-n+1}\right)$ with $k \geqslant g(n)$ until finding the first such interval with $p \notin\left(q_{k}-2^{-n+1}, q_{k}+2^{-n+1}\right)$. Such an interval 
will be found because $\left|q_{k}-\alpha\right|<|p-\alpha|$ for almost all $k$ since $p \neq \alpha$ and $\left(q_{i}\right)_{i}$ converges to $\alpha$.

Then $p \in\left\{q_{i}\right\}$ if and only if $p \in\left\{q_{0}, \ldots, q_{k-1}\right\}$.

(2) $\alpha$ is rational and $\alpha \in\left\{q_{i}\right\}$.

To decide $p \in\left\{q_{i}\right\}$ perform the following procedure:

Check whether $p=\alpha$. If yes $\Gamma$ conclude $p \in\left\{q_{i}\right\}$. If $p \neq \alpha$ then carry out the procedure in (1). The same argument as in (1) applies.

(3) $\alpha$ is rational and $\alpha \notin\left\{q_{i}\right\}$.

To decide $p \in\left\{q_{i}\right\}$ perform the following procedure:

Check whether $p=\alpha$. If yes $\Gamma$ conclude $p \notin\left\{q_{i}\right\}$. If $p \neq \alpha$ then carry out the procedure in (1). The same argument as in (1) applies.

Remark 5. The procedure in the last proof is not uniform in the sequence $\left(q_{i}\right)_{i}$ and a modulus of convergence $g$. Indeed $\Gamma$ a uniform procedure does not exist as one sees by considering the following list of sequences: $\left(r_{i}^{(j)}\right)_{i}$ for $j=0,1,2, \ldots \Gamma$ where $r_{i}^{(j)}=0$ for all $i \in \mathbb{N}$, if $j \in K$, and $r_{i}^{(j)}=\frac{1}{i+1}$ for all $i \in \mathbb{N}$, if $j \notin K$.

The next situation to consider is what happens if we relax the condition that the sequence should converge computably. Which reals do we get as limits of computable sequences $\left(q_{i}\right)_{i}$ and which degrees do we get as degrees of $\left\{q_{i}\right\}$ ?

Proposition 6. For a real $\alpha \in[0,1]$ the following two conditions are equivalent:

1. There exists a computable sequence $\left(q_{i}\right)_{i}$ of rationals converging to $\alpha$.

2. $\alpha=0 . \chi_{A}$ for some $\Delta_{2}^{0}$ set $A$.

Proof. $(\rightarrow)$ We can assume that all rationals $q_{i}$ lie in the unit interval $[0,1]$. Define $x \in A[s]$ if and only if $x<s$ and $q_{s}(x)=1$. Then $A=\lim _{s} A[s]$ is a $\Delta_{2}^{0}$ set and $\alpha=0 . \chi_{A}$.

$(\leftarrow)$ Suppose $\alpha=0 \cdot \chi_{A}$ where $A$ is a $\Delta_{2}^{0}$ set and $\{A[s]\}_{s \in \mathbb{N}}$ is a computable approximation to $A$. Let $q_{i}=0 . \chi_{A[s]}$. Then clearly $\left(q_{i}\right)_{i}$ is a computable sequence converging to $\alpha$.

Furthermore $\Gamma$ if $\alpha=0 . \chi_{A}$ for $A$ a $\Delta_{2}^{0}$ set $\Gamma$ then we can code every c.e. Turing degree into a computable sequence of rationals converging to $\alpha$. 
Theorem 7. Suppose $\alpha=0 . \chi_{A}$ for a $\Delta_{2}^{0}$ set $A$. Then for every c.e. degree $\boldsymbol{b}$ there exists a computable sequence $\left(q_{i}\right)_{i}$ with limit $\alpha$ such that $\left\{q_{i}\right\}$ has degree $b$.

Proof. Let $\left(p_{i}\right)_{i}$ be a computable sequence converging to $\alpha$ such that $\left\{p_{i}\right\}$ is infinite. We can construct a computable subsequence $\left(r_{j}\right)_{j}$ of $\left(p_{i}\right)_{i}$ such that $\theta\left(r_{j}\right)$ is strictly increasing. Let $B$ be an arbitrary infinite c.e. set of natural numbers and $b_{0}, b_{1}, b_{2}, \ldots$ be an effective injective enumeration of $B$. Then the sequence $\left(q_{i}\right)_{i}=\left(r_{b_{i}}\right)_{i}$ is a computable sequence of rationals $\Gamma$ it converges to $\alpha \Gamma$ and we claim that $\left\{q_{i}\right\} \equiv_{T} B$. Indeed $\Gamma$ a natural number $m$ is in $B$ iff $r_{m}$ is in $\left\{q_{i}\right\}$. Conversely $\Gamma$ for an arbitrary rational number $s$ we can decide $s \in\left\{q_{i}\right\}$ by first asking whether $s \in\left\{r_{i}\right\}$. This is decidable because $\theta\left(r_{i}\right)$ is strictly increasing. If the answer is positive we compute the unique number $b$ with $r_{b}=s$ Tand ask whether $b \in B$.

So far we have considered arbitrary computable sequences of rationals that converge. ${ }^{2}$ As we remarked in the introduction $\Gamma$ if the left cut $L(\alpha)$ is c.e. and $\alpha=0 . \chi_{A}$ then $A$ may not be a c.e. set. By restricting our attention to increasing computable sequences we make the following definition of computably enumerable real.

Definition 8. A real $\alpha$ is computably enumerable if there is an increasing computable sequence of rationals converging to $\alpha$.

In Theorem 10 below we give a characterization of the c.e. reals. and classify those sets $A$ for which $\alpha=0 . \chi_{A}$ has $L(\alpha)$ c.e. We first motivate a definition that is useful for us.

Suppose $\left(q_{i}\right)_{i}$ is an increasing computable sequence of rationals in $[0,1]$ converging to $\alpha=0 . \chi_{A}$. There is a natural computable approximation to $A$ derived from $\left(q_{i}\right)_{i}$, namely $A=\lim _{s} A[s]$ where $A[s]=\left\{n \mid n<s \& q_{s}(n)=1\right\}$. Of course each $A[s]$ is a finite set. Furthermore $\Gamma$ if $x \in A[s]$ but $x \notin A[s+1]$ then because $\left(q_{i}\right)_{i}$ is an increasing sequence there must be some $y<x$ such that $y \in A[s+1] \backslash A[s]$.

Definition 9. Let $A$ be a $\Delta_{2}^{0}$ set. We say that $A$ is strongly $\omega$-c.e if there is a computable approximation $(A[s])_{s}$ to $A$ such that

1. $A[0]=\emptyset$,

2. $x \in A[s] \backslash A[s+1] \Longrightarrow \exists y<x(y \in A[s+1] \backslash A[s])$.

Then the following theorem characterises the c.e. reals.

\footnotetext{
${ }^{2}$ For further consideration of computable sequences having a noncomputable modulus of convergence, the interested reader should consult Calude and Hertling [3].
} 
Theorem 10 (Calude, Hertling, Khoussainov and Wang [4]). The following conditions are equivalent for a real $\alpha \in[0,1]$.

1. $\alpha$ is a c.e. real.

2. $L(\alpha)$ is c.e.

3. There is a strongly $\omega$-c.e. set $A$ such that $\alpha=0 . \chi_{A}$.

Proof. $(2) \rightarrow(1)$. Suppose $L(\alpha)$ is c.e. Then it is easy to generate an increasing computable sequence of rationals from an effective enumeration of $L(\alpha)$.

$(1) \rightarrow(3)$. The assertion holds for $\alpha=0$. Suppose $\alpha>0$ and that $\left(q_{i}\right)_{i}$ is an increasing computable sequence of rationals in $[0,1]$ converging to $\alpha$. Define $A=\lim _{s} A[s]$ where $A[s]=\left\{x \mid x<s \& q_{s}(x)=1\right\}$. Then of course $\alpha=0 . \chi_{A}$. It is also clear that $A$ is strongly $\omega$-c.e.

$(3) \rightarrow(2)$. Let $\alpha=0 . \chi_{A}$ for some strongly $\omega$-c.e. set $A$. Let $q_{s}=0 . \chi_{A[s]}$ where $\{A[s]\}_{s \in \mathbb{N}}$ is a computable approximation to $A$ satisfying Definition 9 . Then $L(\alpha)$ can be enumerated from an enumeration of $\left\{q_{s} \mid s \in \mathbb{N}\right\}$.

Corollary 11. If $A$ is a strongly $\omega$-c.e. set then $A$ is of c.e. degree.

Proof. We have already mentioned that $L\left(0 . \chi_{A}\right) \equiv_{T} A$ for $A \subseteq \mathbb{N}$. This together with Theorem 10 gives the assertion.

\section{Representations of computably enumerable reals}

For the remainder of the paper we only consider c.e. reals.

Definition 12. A set $B \subseteq \mathbb{Q}$ of rationals is called a representation of $\alpha$ if there is an increasing computable sequence $\left(q_{i}\right)_{i}$ of rationals with limit $\alpha$ and $\left\{q_{i}\right\}=B$. We identify $B$ with $\theta(B)$, its image under the bijection $\theta: \mathbb{Q} \mapsto \mathbb{N}$ and also call $\theta(B)$ a representation of $\alpha$.

We look at the Turing degrees of representations of c.e. reals. As we noted in the introduction $\Gamma \operatorname{deg}_{T}(\alpha)=\operatorname{deg}_{T}(L(\alpha))$.

Lemma 13. Every c.e. degree is the degree of $L(\alpha)$ for some c.e. real $\alpha$.

Proof. Let $A$ be a c.e. set of degree $\boldsymbol{a}$ and let $\alpha$ be the c.e. real equal to $0 . \chi_{A}$. Then it is clear that $L(\alpha) \equiv_{T} A$. 
We direct the reader to Soare [13] for related work on the relative computability of cuts of arbitrary reals.

It turns out that the question about the Turing degrees of c.e. reals is intimately connected with the splitting properties of the c.e. Turing degrees. We first recall some notions from computability theory. (See Downey and Stob [5] for a survey of splitting theorems.)

Definition 14. A splitting of a c.e. set $A$ is a pair of disjoint c.e. sets $A_{1}$ and $A_{2}$ such that $A_{1} \cup A_{2}=A$. Then we say that $A_{1}$ and $A_{2}$ form a splitting of $A$ and that each of the sets $A_{1}$ and $A_{2}$ is a half of a splitting of $A$.

It is easy to see that if $A_{1}$ and $A_{2}$ form a splitting of a c.e. set $A \Gamma$ then $A \equiv_{T} A_{1} \oplus A_{2}$. The following two lemmata show the connection between representations of c.e. reals and splitting.

Lemma 15. If $B$ is a representation of a c.e. real $\alpha$, then $B$ is an infinite half of a splitting of $L(\alpha)$.

Proof. It is clear that any representation $B$ of a c.e. real $\alpha$ is an infinite c.e. subset of $L(\alpha)$. We have to show that also $L(\alpha) \backslash B$ is c.e. Let $\left(q_{i}\right)_{i}$ be the increasing (computable!) sequence of rationals with $B=\left\{q_{i}\right\}$. The set $L(\alpha)$ is c.e. We can for each element $p \in L(\alpha)$ wait until we find a $q_{j}$ with $p \leqslant q_{j}$ (as rationals) $\Gamma$ and choose $p$ if and only if $p \notin\left\{q_{0}, \ldots, q_{j}\right\}$. Hence $\Gamma$ we can enumerate $L(\alpha) \backslash B$.

Lemma 16. Let $B$ be a representation of a c.e. real $\alpha$. For a subset $C \subseteq B$ the following two conditions are equivalent:

1. $C$ is a representation of $\alpha$.

2. $C$ is an infinite half of a splitting of $B$.

Proof. $(\rightarrow)$ As the proof of Lemma 15 .

$(\leftarrow)$ Let $\left(q_{i}\right)_{i}$ be the increasing computable sequence of rationals with $B=\left\{q_{i}\right\}$ Tlet $C$ be an infinite half of a splitting of $B$ Cand let $D$ be the other half of this splitting. We construct an increasing rational sequence $\left(p_{i}\right)_{i}$ with limit $\alpha$ and $C=\left\{p_{i}\right\}$ by going through the list $\left(q_{i}\right)_{i} \Gamma$ by waiting for each element $q_{i}$ until it is enumerated either in $C$ or in $D \Gamma$ and by choosing it if and only if it is enumerated in $C$.

It follows from Lemma 15 that $L(\alpha)$ possesses the greatest degree amongst representations of $\alpha$

Corollary 17. If $B$ is a representation of a c.e. real $\alpha$, then $B \leqslant_{T} L(\alpha)$.

For the special case of computable reals we then get the following: 
Corollary 18. If $\alpha$ is a computable real, then every representation of $\alpha$ is computable.

We consider the following partial orders.

Definition 19. For a c.e. real $\alpha$ let $\mathcal{L}(\alpha)$ be the partial order (with respect to Turing reducibility) of those c.e. Turing degrees below $\operatorname{deg}_{T}(L(\alpha))$ that contain a representation of $\alpha$.

Proposition 20. For every c.e. real $\alpha, \mathcal{L}(\alpha)$ is an upper-semi lattice.

Proof. Let $\alpha$ be a c.e. real. Then $\mathcal{L}(\alpha)$ is is closed under the usual join operation on Turing degrees. Indeed suppose $\boldsymbol{a}, \boldsymbol{b} \in \mathcal{L}(\alpha)$ with $A$ and $B$ being representations of $\alpha$ in $\boldsymbol{a}$ and $\boldsymbol{b}$ Trespectively. Let $C=A \cup B$. Then $C$ is the representation of $\alpha$ formed by effectively enumerating the two sequences of $A$ and $B$ in increasing order as rationals. We claim that $\operatorname{deg}_{T}(C)=\boldsymbol{a} \cup \boldsymbol{b}$, that is $\theta(C) \equiv_{T} \theta(A) \oplus \theta(B)$. It is obvious that $\theta(C) \leqslant_{T} \theta(A) \oplus \theta(B)$. For the converse we observe that by Lemma $16(\rightarrow)$ the set $A$ is a half of a splitting of $C$ Thence $\theta(A) \leqslant_{T} \theta(C) \Gamma$ the same for $B$.

We now study this upper-semi lattice further. We first prove that $\boldsymbol{O}$ $\operatorname{deg}_{T}(L(\alpha))$ are in $\mathcal{L}(\alpha)$.

Proposition 21. For any c.e. real $\alpha$ there is a computable representation of $\alpha$.

Proof. Take an arbitrary representation of $\alpha$. The classical result that every infinite c.e. set contains an infinite computable subset yields the assertion.

Furthermore we can construct a noncomputable representation as follows.

Theorem 22. Every noncomputable c.e. real $\alpha$ has a noncomputable representation.

Proof. Fix an increasing computable sequence $\left(q_{i}\right)_{i}$ converging to $\alpha$ such that $\left\{q_{i}\right\}$ is computable. We construct a noncomputable representation $B$ such that $\left(p_{i}\right)_{i}$ is a subsequence of $B$ and $B$ is not the complement of every c.e. set.

At stage $s=0$ let $b_{0}=q_{0}$.

At stage $s+1$ we have already constructed $B[s]=\left\{b_{0}, \ldots, b_{k_{s}}\right\}$ where $b_{0}<\ldots<b_{k_{s}}$ (as rationals) and $b_{k_{s}}=q_{s}$.

If there is a least $e<s+1$ such that $W_{e}[s] \cap B[s]=\emptyset$ and an $x \in W_{e}[s]$ with $q_{s}<x \leqslant q_{s+1}$ then let $b_{k_{s}+1}=x, b_{k_{s}+2}=q_{s+1}$ and $k_{s+1}=k_{s}+2$.

If there is no such $e$ then let $b_{k_{s}+1}=q_{s+1}$ and $k_{s+1}=k_{s}+1$.

We complete the construction by letting $B=\bigcup_{s} B[s]$. 
Clearly $\left(b_{i}\right)_{i}$ is an increasing computable sequence of rationals converging to $\alpha$. It remains to show that $B$ is not computable.

Suppose $B$ is a computable set. Then let $e$ be the least index such that $B=\overline{W_{e}}$. Let $s_{0}$ be a stage such that for all $i<e$ and all $s \geqslant s_{0}$ we have $W_{i}[s] \cap B[s] \neq \emptyset$ or there is no $x \in W_{i}[s]$ with $q_{s}<x \leqslant q_{s+1}$. We will show that for all $p>q_{s_{0}}$ (as rationals) $\Gamma p \in L(\alpha)$ is decidable $\Gamma$ contradicting the hypothesis of the theorem. To compute $p \in L(\alpha)$, enumerate $B$ and $W_{e}$ until $p$ occurs in one of them. If $p \in B$ then $p \in L(\alpha)$. Otherwise $p \in W_{e}$ and we claim that $p \notin L(\alpha)$. For suppose $p \in L(\alpha)$, then at some least stage $t>s$, $q_{s_{0}}<p \leqslant q_{t}$, and the construction enumerates some $p^{\prime} \in B$ for $q_{s_{0}}<p^{\prime} \leqslant q_{t}$ and $p^{\prime} \in W_{e}$. This contradicts $B \cap W_{e}=\emptyset$ and hence $B$ is not a computable set.

We now show that $\operatorname{deg}_{T}(L(\alpha))$ is in the lattice.

Theorem 23. Let $\alpha$ be a c.e. real. Then $\alpha$ has a representation of degree $L(\alpha)$. Furthermore, every representation of $\alpha$ can be extended to a representation of degree $L(\alpha)$.

Proof. Let $\left(p_{i}\right)_{i}$ be an increasing computable sequence of rationals converging to $\alpha$. We shall construct a new computable sequence $\left(q_{i}\right)_{i}$ of rationals such that $\left\{q_{i}\right\}$ is a representation of $\alpha$ with $\left\{q_{i}\right\} \equiv_{T} L(\alpha)$. Additionally we define $l_{i}=\max \left\{\theta\left(p_{j}\right) \mid j \leqslant i\right\}$ for all $i \Gamma$ and we will define a sequence $\left(j_{i}\right)_{i}$ of natural numbers with $q_{j_{i}}=p_{i}$ for all $i$. We start with $j_{0}=0$ and $q_{0}=p_{0}$. Given $j_{i}$ with $q_{j_{i}}=p_{i}$ Twe define $j_{i+1}>j_{i}$ such that

$$
j_{i+1}-j_{i}=\left|\left\{q \in \mathbb{Q} \mid p_{i}<q \leqslant p_{i+1} \& \theta(q) \leqslant l_{i+1}\right\}\right|
$$

and for $m=1, \ldots, j_{i+1}-j_{i}$ we define the numbers $q_{j_{i}+m}$ as the rational numbers in this set in increasing order.

It is obvious that $\left(q_{i}\right)_{i}$ is an increasing computable sequence of rationals converging to $\alpha \Gamma$ and $q_{j_{i}}=p_{i}$ for all $i$. From Corollary 17 we know $\left\{q_{i}\right\} \leqslant_{T} L(\alpha)$. We still have to prove $L(\alpha) \leqslant_{T}\left\{q_{i}\right\}$. Let $p \in \mathbb{Q}$. In order to decide $p \in L(\alpha)$ we compute the minimal $k$ with $l_{k} \geqslant \theta(p)$. Then we check whether $p \leqslant q_{j_{k}}$. If $p \leqslant q_{j_{k}}$ Tthen clearly $p \in L(\alpha)$. If $p>q_{j_{k}}$ Tthen $p \in L(\alpha)$ if and only if $p \in\left\{q_{i}\right\}$.

We give an alternative proof by a slighty different construction. It shows that we can obtain a representation of $\alpha$ of degree $L(\alpha)$ consisting only of dyadic rational numbers.

Fix an increasing computable sequence $\left(p_{i}\right)_{i}$ of dyadic rationals with limit $\alpha$ with increasing denominator:

$$
p_{i}=\frac{2 n_{i}+1}{2^{k_{i}}}
$$


for a computable sequence $\left(n_{i}\right)_{i}$ of integers and a computable $\Gamma$ increasing sequence $\left(k_{i}\right)_{i}$ of natural numbers. We shall construct a new computable sequence $\left(q_{i}\right)_{i}$ of rationals such that $\left\{q_{i}\right\}$ is a representation of $\alpha$ having Turing degree $\operatorname{deg}_{T}(L(\alpha))$. We will define a sequence $\left(j_{i}\right)_{i}$ of natural numbers. We will have $q_{j_{i}}=p_{i}$ for all $i$.

We start with $j_{0}=0$ and $q_{0}=p_{0}$. Given $j_{i}$ with $q_{j_{i}}=p_{i}$ Twe set

$$
q_{j_{i}+m}=q_{j_{i}}+\frac{m}{2^{k_{i+1}}}
$$

for $m=1, \ldots,\left(p_{i+1}-p_{i}\right) \cdot 2^{k_{i+1}}$ and

$$
j_{i+1}=j_{i}+\left(p_{i+1}-p_{i}\right) \cdot 2^{k_{i+1}} .
$$

Of course $\Gamma\left(q_{i}\right)_{i}$ is an increasing computable sequence of rationals converging to $\alpha$ since $q_{j_{i}}=p_{i}$ for all $i$.

We have to show $L(\alpha) \leqslant_{T}\left\{q_{i}\right\}$. If $\alpha$ is a rational then $L(\alpha)$ is computable and hence $\leqslant_{T}\left\{q_{i}\right\}$. So we assume that $\alpha$ is irrational.

The important property of the set $\left\{q_{i}\right\}$ is that if it contains a dyadic number $\frac{2 n+1}{2^{k}} \Gamma$ then it contains all dyadic numbers in the interval $\left(\frac{2 n+1}{2^{k}}, \alpha\right)$ whose denominator is at most $2^{k}$. But $\left\{q_{i}\right\}$ does not contain any number greater than $\alpha$. Furthermore $\Gamma$ the denominator of the dyadic number $q_{j_{i}}$ is at least $2^{k_{i}} \geqslant 2^{i}$. Hence $\Gamma$ given $\left\{q_{i}\right\}$ as an oracle $\Gamma$ for an arbitrary natural number $l$ we can compute a dyadic rational $\frac{2 n+1}{2^{k}}$ with $k \geqslant l$ and such that the interval $\left(\frac{2 n+1}{2^{k}}, \frac{2 n+3}{2^{k}}\right)$ contains $\alpha$. Using $\left\{q_{i}\right\} \Gamma$ for a given rational number $r$, we can decide whether $r<\alpha$ by computing such an interval which contains $\alpha$ but not $r$ (any sufficiently small interval containing the irrational number $\alpha$ will not contain $r$ ) and checking whether $r$ lies to the left or to the right of this interval.

Corollary 24. Every c.e. degree contains a representation of a c.e. real.

Proof. By Lemma 13 and Theorem 23.

We can strengthen Lemma 15 by using the method of proof from Theorem 23 .

Theorem 25. Suppose $B$ is a representation of a noncomputable c.e real $\alpha$. Then there is a representation $C$ of $\alpha$ such that $C \equiv_{T} L(\alpha) \backslash B$.

Proof. Let $\left(b_{i}\right)$ be the increasing computable sequence such that $B=\left\{b_{i}\right\}$. Let $\left(p_{i}\right)_{i}$ be a representation of $\alpha$ such that $\left\{p_{i}\right\}$ is computable and $\left\{p_{i}\right\} \cap\left\{b_{i}\right\}=\emptyset$. We construct a new increasing computable sequence of rationals $\left(c_{i}\right)_{i}$ such that $\left\{c_{i}\right\} \equiv_{T} L(\alpha) \backslash B$. Define $l_{i}=\max \left\{\theta\left(p_{j}\right) \mid j \leqslant i\right\}$ for all $i$, and we will define a sequence $\left(j_{i}\right)_{i}$ of natural numbers with $c_{j_{i}}=p_{i}$ for all $i$. We start with 
$j_{0}=0$ and $c_{0}=p_{0}$. Let $b_{p_{i}}$ denote the least rational in $B$ which is greater than $p_{i}$. Then given $j_{i}$ with $c_{j_{i}}=p_{i}$, define $j_{i+1}>j_{i}$ such that

$$
j_{i+1}-j_{i}=\left|\left\{q \in \mathbb{Q} \mid p_{i}<q \leqslant p_{i+1} \& \theta(q) \leqslant l_{i+1} \& q \notin\left\{b_{0}, \ldots, b_{p i+1}\right\}\right\}\right|,
$$

and for $m=1, \ldots, j_{i+1}-j_{i}$ we define $c_{j_{i}+m}$ to be those rational numbers in this set in increasing order. Let $C=\left\{c_{i}\right\}$.

It is clear that $\left(c_{i}\right)_{i}$ is an increasing computable sequence of rationals converging to $\alpha$, since $c_{j_{i}}=p_{i}$ for all $i$. We now show that $C \equiv_{T} L(\alpha) \backslash B$.

First $\Gamma C \leqslant_{T} L(\alpha) \backslash B$ as follows. Let $p \in \mathbb{Q}$. If $p \notin L(\alpha) \backslash B$ then $p \notin C$. Otherwise $\Gamma$ if $p \in L(\alpha) \backslash B$, enumerate $C$ until reaching a least $c_{i}$ such that $c_{i} \geqslant p$. Then $p \in C$ if and only if $p \in\left\{c_{0}, \ldots, c_{i}\right\}$.

Secondly $\Gamma L(\alpha) \backslash B \leqslant_{T} C$ as follows. Let $p \in \mathbb{Q}$. Compute the least $k$ such that $l_{k} \geqslant \theta(p)$ and then check whether $p \leqslant c_{j_{k}}$. If $p \leqslant c_{j_{k}}$ then enumerate $B$ until reaching a least $b_{i}$ such that $p \leqslant b_{i}$, and conclude $p \in L(\alpha) \backslash B$ if and only if $p \notin\left\{b_{0}, \ldots, b_{i}\right\}$. Otherwise $\Gamma p>c_{j_{k}}$ and we can conclude that $p \in L(\alpha)$ if and only if $p \in C$.

So we have established that for noncomputable c.e. reals $\alpha,|\mathcal{L}(\alpha)| \geqslant 2$. Are there intermediate representations? That is $\Gamma$ for every noncomputable c.e. real $\alpha$, is there a representation $B$ such that $\emptyset<_{T} B<_{T} L(\alpha)$ ? We call upon a classical result from computability theory; see Soare [14ГChapter VII.3] for more details.

Theorem 26 (Sacks Splitting Theorem). Let $A$ and $D$ be given noncomputable c.e. sets. Then there are low c.e. sets $B$ and $C$ such that $A=B \cup C$, $B \cap C=\emptyset$ and $D \mathbb{K}_{T} B, C$.

Corollary 27. Let $\alpha$ be a noncomputable c.e. real and let $D$ be a noncomputable c.e. set. Then there is a noncomputable representation $B$ of $\alpha$ such that $D \mathbb{K}_{T} B$ and $B$ is low.

Proof. By Theorem 23 there exists a noncomputable representation $A$ of $\alpha$. Apply Sacks Splitting Theorem to $A$ and $D$. At least one of the obtained sets $B$ and $C$ is noncomputable $\Gamma$ hence also infinite and by Lemma $16(\leftarrow)$ a representation of $\alpha$.

Remark 28. It is possible to construct directly a low noncomputable representation $B$ avoiding the upper cone of a c.e. $D$. This is done via a finite priority argument combining the construction of Theorem 22 with the usual techniques of Sacks restraint and lowness requirements. See Soare [14ГChapter VII] VII for these techniques.

So how many representations do noncomputable c.e. reals have? 
Corollary 29. Let $\alpha$ be a noncomputable c.e. real and $A$ a noncomputable representation of $\alpha$. Then there is a low representation $B$ of $\alpha$ such that $\emptyset<_{T} B<_{T} A$.

Proof. Apply Corollary 27 with $D=A$.

Repeated application of this corollary shows that that every noncomputable c.e. real has infinitely many representations of different degree $\Gamma$ and in fact the representations of noncomputable c.e. reals are downwards dense in the Turing degrees.

\section{The cone below $L(\alpha)$}

In the light of the above results it is natural to ask whether every c.e. degree in the cone below $L(\alpha)$ contains a representation of $\alpha$. We say that $\alpha$ realises the cone if for all c.e. sets $A \leqslant_{T} L(\alpha)$ there is a computable increasing sequence of rationals converging to $\alpha$ of degree $A$.

Once more $\Gamma$ splitting properties of the c.e. Turing degrees play a role. Let

$$
S(A)=\left\{\boldsymbol{c} \mid \exists A_{1}\left(A_{1} \text { is half of a splitting of } A \text { and } \operatorname{deg}_{T}\left(A_{1}\right)=\boldsymbol{c}\right)\right\} .
$$

Definition 30. A computably enumerable set $A$ has the Universal Splitting Property if $S(A)=\left\{\boldsymbol{b} \mid \boldsymbol{b} \leqslant \operatorname{deg}_{T}(A)\right\}$. $A$ is non-USP otherwise.

Lerman and Remmel were motivated to study this property from investigations by Remmel in effective algebra.

Theorem 31 (Lerman and Remmel $[7,8])$. There is a c.e. degree $\boldsymbol{a}$ such that every c.e. set of degree $\boldsymbol{a}$ is non-USP.

Such a degree is called completely non-USP.

Theorem 32. There is a noncomputable c.e. real $\alpha$ that does not realise the cone.

Proof. By Theorem 31 take a c.e. degree $\boldsymbol{a}$ which is completely non-USP. Let $\alpha$ be a c.e. real such that $L(\alpha) \in \boldsymbol{a}$. Since $\boldsymbol{a}$ is completely non-USP $\Gamma$ there is a c.e. degree such that $\nvdash<\boldsymbol{b}<\boldsymbol{a}$ and $\boldsymbol{b}$ contains no half of a splitting of $L(\alpha)$. Now suppose $\left\{q_{i}\right\}$ is a representation of $\alpha$. By Lemma $15\left\{q_{i}\right\}$ is half of a splitting of $L(\alpha)$, and hence cannot have Turing degree $\boldsymbol{b}$.

It is possible to construct directly a noncomputable c.e. real that does not realise the cone. We conclude this section by sketching the proof in considerable detail. 
We construct a noncomputable c.e. real $\alpha$, a c.e. set $B$ and a Turing functional $\Gamma$ to meet the following requirements for all Turing functionals $\Phi$ and $\Psi$ and c.e. sets $W$ :

$\mathrm{R}: B=\Gamma(L(\alpha))$

$\mathrm{P}_{\Phi}: B \neq \Phi$

$\mathrm{N}_{\Phi, \Psi, W}: B=\Phi(W) \wedge W=\Psi(B) \Longrightarrow W \not \rightarrow \alpha$.

The notation $W \not \rightarrow \alpha$ denotes that $\Gamma$ when viewed as a computable sequence of rationals $\Gamma W$ is either not increasing $\Gamma$ or else does not converge to $\alpha$.

In the R-requirement we really mean $\theta(L(\alpha))$ but this is cumbersome and so we identify $L(\alpha)$ with $\theta(L(\alpha))$.

We construct $\alpha$ by stages so that $\alpha_{0}<\alpha_{1}<\ldots<\alpha_{s}<\ldots$ is a computable increasing sequence of rationals converging to $\alpha$. This also gives us an enumeration of $L(\alpha)$. To ensure convergence we insist that $\alpha_{i}<1$ for all $i \in \mathbb{N}$. Note that the $\mathrm{R}$ requirement together with all the $\mathrm{P}_{\Phi}$ requirements ensure that $L(\alpha)$ is not computable and so $\alpha$ will be a noncomputable c.e. real.

As usual we order the $\mathrm{P}$ and $\mathrm{N}$ requirements in some computable way of order type $\omega$. Let $\mathrm{S}$ denote a general $\mathrm{P}$ or $\mathrm{N}$ strategy. The strategies for meeting the $\mathrm{P}$ and $\mathrm{N}$ requirements will mean imposing a restriction on the construction of $\alpha$. To restrain $\alpha<p$ for some $p \in \mathbb{Q}$ for a strategy $\mathrm{S}$ is to prevent lower priority strategies than $\mathrm{S}$ from ever defining $\alpha \geqslant p$ while the restraint is in place. Notice that if $\alpha$ is restrained less than $p$ for almost all stages of the construction $\Gamma$ then $\alpha \neq p$ since $\alpha$ is constructed to be a noncomputable c.e. real. The $\mathrm{R}$ requirement is a global requirement and acts as described below.

The strategy for R. We must construct $\Gamma$ such that $B=\Gamma(L(\alpha))$. So for every $x \in \mathbb{N}$ we have a trace $\gamma(x) \in \mathbb{N}$ such that $B(x)=\Gamma\left(L(\alpha) \uparrow_{\gamma(x)+1}\right)(x) \downarrow$. That is $\Gamma$ for each $x, \Gamma$ correctly computes $B(x)$ using an initial segment of the oracle $L(\alpha)$. The $\mathrm{R}$ strategy acts at the end of each stage $s+1$ and for each $x<s+1$ with $\Gamma(L(\alpha))(x) \uparrow$, it defines $\Gamma\left(L(\alpha)\left\lceil_{\gamma(x)[s+1]+1}\right)(x) \downarrow=B(x)[s+1]\right.$.

To ensure that $\Gamma$ is consistent we must never define $\Gamma\left(L(\alpha) \uparrow_{\gamma(x)+1}\right)(x)=1$ and $\Gamma\left(L(\alpha) \Gamma_{\gamma(x)+1}\right)(x)=0$.

The strategies for $\mathrm{P}$ and $\mathrm{N}$ requirements will involve the selection of witnesses $x$ that may be enumerated into $B$ at some stage. Note that there will be some numbers $x$ that are never chosen as witnesses for the strategies for the $\mathrm{N}$ and $\Phi$ requirements. For such numbers we can define $\gamma(x)=0$ and $\Gamma\left(L(\alpha) \uparrow_{\gamma(x)+1}\right)(x) \downarrow=0=B(x)$.

So suppose $x$ is chosen as a witness at stage $s_{0}$ for some strategy $\Gamma \mathrm{S}$ say. Initially $x \notin B$. When we choose $x$ at stage $s$ say $\Gamma$ we also choose some trace 
$\gamma(x)[s] \in \mathbb{N}$ currently not in $L(\alpha)[s]$ and define

$$
\Gamma\left(L(\alpha)\left\lceil_{\gamma(x)[s]+1}\right)(x) \downarrow=0 .\right.
$$

The choice of value for $\gamma(x)[s]$ is important for the following reason. Typically we do not know whether $x$ is enumerated into $B$ by its strategy. If $x$ is enumerated into $B$ at some stage $t+1>s$ then we must correct

$$
\Gamma\left(L(\alpha)\left\lceil_{\gamma(x)[s]+1}\right)(x) \downarrow=1=B(x) .\right.
$$

The only way to do this and keep $\Gamma$ consistent is to change $L(\alpha)[s]\left\lceil_{\gamma(x)[s]+1}\right.$ by enumerating $\gamma(x)[s]$ into $L(\alpha)$, which corresponds to defining $\alpha_{t+1} \geqslant \gamma(x)[s]$. So when we choose $\gamma(x)[s]$ we insist that all requirements of lower priority than $\mathrm{S}$ do not define $\alpha_{t^{\prime}} \geqslant \theta^{-1}(\gamma(x)[s])$ at each stage $t^{\prime}>s$ for which $x \notin B\left[t^{\prime}\right]$.

Because we cannot know in advance which witnesses $x$ enter $B$, while $\mathrm{S}$ restrains $\alpha$ to the left of $\gamma(x)[s]$, a lower priority strategy $\mathrm{S}^{\prime}$ must assign a trace $\gamma\left(x^{\prime}\right)\left[s^{\prime}\right]$ for its witness $x^{\prime}$ such that $\theta^{-1}\left(\gamma\left(x^{\prime}\right)\left[s^{\prime}\right]\right)<\theta^{-1}(\gamma(x)[s])$. This means that $\mathrm{S}^{\prime}$ can enumerate its witness $x^{\prime}$ into $B$ at some stage $s_{0}^{\prime}$, enumerate $\gamma\left(x^{\prime}\right)\left[s^{\prime}\right]$ into $L(\alpha)$ to correct

$$
\Gamma\left(L(\alpha) \uparrow_{\gamma\left(x^{\prime}\right)\left[s^{\prime}\right]+1}\right)\left(x^{\prime}\right) \downarrow=1=B\left(x^{\prime}\right),
$$

by defining $\gamma\left(x^{\prime}\right)\left[s^{\prime}\right] \leqslant \alpha_{s_{0}^{\prime}}<\gamma(x)[s]$. Further $\Gamma$ if at some later stage $s_{0}$ say $\Gamma \mathrm{S}$ wants to enumerate $x$ into $B$ it may do so as there is still the possibility to correct $\Gamma$ on argument $x$ by enumerating $\gamma(x)[s]$ into $L(\alpha)$.

We may increase the value of $\gamma(x)[s]$ occasionally $\Gamma$ but will do so only finitely often so that $\Gamma$ total for oracle $L(\alpha)$.

With the above in mind we now discuss the strategies for $\mathrm{P}$ and $\mathrm{N}$ requirements.

The Strategy for $\mathrm{P}_{\Phi}$. This strategy is essentially the usual one. We simultaneously assume the presence of $\mathrm{R}$.

Choose a witness $x$ currently not in $B\left[s_{0}\right]$ and a trace $\gamma(x)\left[s_{0}\right]$. The $\mathrm{R}$ strategy will define $\Gamma\left(L(\alpha) \uparrow_{\gamma(x)\left[s_{0}\right]+1}\right) \downarrow=0$ at the end of stage $s_{0}$. This imposes a restraint on lower priority requirements on defining $\alpha<\theta^{-1}\left(\gamma(x)\left[s_{0}\right]\right)$ as discussed above. Now wait for $\Phi(x) \downarrow[s]=0$ at some stage $s$. Then enumerate $x \in B[s]$ and define $\alpha_{s} \geqslant \gamma(x)\left[s_{0}\right]$. This enables the correction of

$$
\Gamma\left(L(\alpha)\left\lceil_{\gamma(x)\left[s_{0}\right]+1}\right)(x)=1=B(x) .\right.
$$

There are two outcomes. Either we wait forever for $\Phi(x) \downarrow=0$ in which case $B(x)=0 \neq \Phi(x)$, or else $\Phi(x) \downarrow=0 \neq 1=B(x)$.

The strategy for $\mathrm{N}_{\Phi, \Psi, W}$. We first introduce some notation and computable functions. A halting computation $\Gamma$ by definition $\Gamma$ can only use a finite amount of resources. Hence we define the use function $\phi$ for a Turing functional $\Phi$ to 
be $\phi(A)(x)[s]=1+$ the largest number used in the computation $\Phi(A)(x)[s]$ if $\Phi(A)(x)[s] \downarrow$. Otherwise $\phi(A)(x)[s]=0$. (For $\Psi$ we use $\psi$ to denote the use function.) We often abbreviate $\phi(A)(x)$ to $\phi(x)$ if it is clear to which oracle we are referring.

We present an itemised strategy annotated with hopefully helpful explanation in parentheses [...]. It is worth bearing in mind the following intuition. The basic idea for this requirement is to approximate the length of agreement between $(B=\Phi(W) \wedge W=\Psi(B))$. When we estimate that agreement between the terms holds beyond some point $x \notin B$, we change $B$ by enumerating in $x$ and hope to force a $W$ change that means $W \not \rightarrow \alpha$. In general we will have to do a little work to arrange that the $W$-change is one that witnesses $W \not \rightarrow \alpha$.

If at any stage we observe that $W$ is not an increasing sequence of rationals then we have trivially satisfied this requirement and the strategy below ceases. Hence from now on we assume $W$ is an enumeration of an increasing sequence of rationals. We begin the strategy at stage $s_{0}$.

1. Choose a witness $x$ larger than any number previously mentioned in the construction (hence not currently in $B$ ) and choose $\gamma(x)\left[s_{0}\right]$ as a trace for $x$.

[The R strategy will define $\Gamma\left(L(\alpha) \uparrow_{\gamma(x)\left[s_{0}\right]+1}\right) \downarrow=0$ at the end of stage $s_{0}$. This imposes a restraint on lower priority requirements on defining $\alpha$ as discussed above in the $\mathrm{R}$ strategy.]

2. Wait for $B \uparrow_{x+1}=\Phi(W) \uparrow_{x+1}$ and $W \uparrow_{\phi(x)+1}=\Psi(B) \uparrow_{\phi(x)+1}$.

[If this never happens then we win the requirement.]

3. Restrain $B \uparrow_{\psi \phi(x)+1}$.

[This restraint is important for steps that follow. We want to control $W$ in some way.]

4. While 2 is satisfied consider those rationals enumerated by $W$ so far $\Gamma$ namely $\theta^{-1}\left(w_{0}\right), \ldots, \theta^{-1}\left(w_{k}\right)$, for some $k \leqslant s$. If $\theta^{-1}\left(w_{i}\right)>\alpha_{s}$ for some least $i, 1 \leqslant i \leqslant k$, then impose a restraint of $\alpha<\theta^{-1}\left(w_{i}\right)$ on lower priority requirements.

[Then $W$ cannot converge to $\alpha$ and be an increasing computable sequence of rationals $\Gamma$ because some member of $W$ is greater than $\alpha$.]

5. Otherwise while $w_{0}, \ldots, w_{t}$ can potentially be an increasing computable sequence converging to $\alpha$. Restrain $\alpha<\theta^{-1}\left(\gamma(x)\left[s_{0}\right]\right)$.

[We may use $x$ as a witness for $\mathrm{N}$, and if we do we will need to correct $\Gamma\left(L(\alpha)\left\lceil_{\gamma(x)\left[s_{0}\right]+1}\right)(x)\right.$ by enumerating $\gamma(x)\left[s_{0}\right]$ into $L(\alpha)$. ] 
6. Consider $Y=\{y \mid y \leqslant \phi(W)(x) \wedge y \notin W[s]\}$.

[The idea is that we want to enumerate $x$ into $B$ to create a disagreement between $B$ and $\Phi(W)$. However $W$ is not under our control $\Gamma$ and so $W \uparrow_{\phi(x)+1}$ may change at some later stage to correct $\Phi(W)(x)=B(x)$. Now $W$ is a c.e. set so can only change by enumerating some number in the set $Y$ above. Therefore we try to create a scenario such that when $W$ changes on an element of $Y, W$ can no longer be an increasing computable sequence converging to $\alpha$.]

Perform the first case below which applies.

(i) For all $y \in Y, \theta^{-1}(y)<\alpha_{s} \vee \theta^{-1}(\gamma(x)[s])<\theta^{-1}(y)$.

[This is our desired situation. In this case $\Gamma$ we are guaranteed that after enumerating $x$ into $B$, if $W$ changes at stage $t$ say to return $B[t] \uparrow_{x+1}=\Phi(W)[t] \uparrow_{x+1}$ and $W[t] \Upsilon_{\phi(x)+1}=\Psi(B)[t] \uparrow_{\phi(x)+1}$, then $W \nrightarrow \rightarrow \alpha$. Cases (ii) and (iii) below deal with arranging that this situation arises.]

(ii) For all $y \in Y, y \neq \gamma(x)[s]$ and $\theta^{-1}(Y) \cap\left[\alpha_{s}, \theta^{-1}(\gamma(x)[s])\right] \neq \emptyset$.

[The problem with this situation is that after enumerating $B$ and defining $\alpha_{s+1} \geqslant \theta^{-1}(\gamma(x)[s]), W$ is able to change on some elements of $Y$ and continue to be potentially a sequence converging to $\alpha$. The action we take in 8 on behalf of this case is to remove such undesired members of $Y$.]

(iii) For some $y \in Y, \theta^{-1}(y)=\theta^{-1}(\gamma(x)[s])$.

[This case prevents the action we take in 8 for the sake of (ii). We would like to remove the undesirable elements of $Y$ Tnamely those in the interval $\left.\left[\alpha_{s}, \theta^{-1}(\gamma(x)[s])\right]\right]$, by increasing $\alpha_{s+1}$ to exceed them all. We cannot do this for the $y$ such that $y=\gamma(x)[s]$ without enumerating $\theta^{-1}(\gamma(x)[s])$ into $L(\alpha)$.]

7. If $6(\mathrm{i})$ occurs then enumerate $x$ into $B$ and let $\alpha_{t+1}=\theta^{-1}(\gamma(x)[s])$. Restrain $\alpha<\theta^{-1}(\tilde{y})$ where

$$
\theta^{-1}(\tilde{y})=\min \left\{\theta^{-1}(y) \mid y \in Y \& \theta^{-1}(y)>\theta^{-1}(\gamma(x)[s])\right\}
$$

[The restraint is make sure that a $W$-change from the set $Y$ guarantees $W \nrightarrow \rightarrow \alpha$ because every member of $Y$ corresponds to a rational greater than $\alpha$ or less than some rational already enumerated by $W$.]

8. If 6 (ii) applies then define $\alpha_{s+1}$ to be the rational $p$ with least $\theta(p)$ such that

$$
p>\left\{\theta^{-1}(y) \mid y \in Y \& \theta^{-1}(y) \in\left[\alpha_{s}, \theta^{-1}(y)(\gamma(x)[s])\right]\right\}
$$


and $p<\theta^{-1}(\gamma(x)[s])$. Return to 6 .

[It was important to insist that no $y \in Y$ was equal $\gamma(x)[s]$, so that we could define $\alpha_{s+1}$ to be greater than the undesirable elements of $Y$ without enumerating $\gamma(x)[s]$ into $L(\alpha)$. Hence $x$ is still our witness and we still have the possibility of enumerating it into $B$ and correcting $\Gamma$ via the enumeration of $\gamma(x)[s]$ into $L(\alpha) \Gamma$ should case $6(\mathrm{i})$ ever occur at a later stage.]

9. If 6 (iii) applies then define $\alpha_{s+1}=\theta^{-1}(\gamma(x)[s])$. Choose a new value for $\gamma(x)[s+1]$ such that $\gamma(x)[s+1] \notin Y$ and $\theta^{-1}(\gamma(x)[s+1])>\alpha_{s+1}$. Restrain $\alpha<\theta^{-1}(\gamma(x)[s])$. Return to 4 .

[By enumerating $\theta^{-1}(\gamma(x)[s])$ into $L(\alpha)$ we cause $\Gamma\left(L(\alpha) \uparrow_{\gamma(x)[s]+1}\right)(x) \uparrow$ and then we can choose a new value for the trace $\gamma(x)[s+1]$. It is important that we only have to do this finitely often so that for the $\Gamma(L(\alpha))$ is total.]

We summarise the outcomes for $\mathrm{N}_{\Phi, \Psi, W}$ :

$w$ : Wait at step 2 or 4 . Then either $B \neq \Phi(W)$ or $W \neq \Psi(B)$, or $W$ is not an increasing sequence of rationals converging to $\alpha$.

$s$ : Stop at 7. Then either $B(x) \neq \Phi(W)(x)$ or $W \neq \Psi(B)$ or else $W$ is not an increasing sequence of rationals.

Note that we cannot loop between 8 and 6 or 9 and 6 infinitely often because having passed through 8 or 9 once $\Gamma$ then $6(\mathrm{i})$ applies and consequently the stratgey stops at step 7 .

Now notice that the strategies for $\mathrm{N}$ and $\mathrm{P}$ above only have finitary outcomes $\Gamma$ and only impose a finite restraint on lower priority requirements. Hence the strategies can be be combined on a tree of strategies in the standard way to form finite injury priority argument to prove the theorem. Whenever a change of outcome is seen for an $\mathrm{N}$ or $\mathrm{P}$ strategy $\mathrm{Call}$ lower priority strategies are initialised and begin from the start. This concludes our discussion.

\section{Open Questions}

We conclude with several open questions concerning the Turing degrees of increasing computable sequences of rationals converging to reals. Again there is a connection with the splitting properties of c.e. sets and to the structure of $S(A)$ for c.e. sets $A$.

1. Is $\mathcal{L}(\alpha)$ dense for all noncomputable c.e. reals $\alpha$ ?

2. Is there a noncomputable c.e. real $\alpha$ that realises the cone? If there is a completely USP c.e. degree $\Gamma$ then the answer is yes. 
3. Are there c.e. reals $\alpha$ and $\beta$ such that $\mathcal{L}(\alpha) \neq \mathcal{L}(\beta)$ ?

4. Characterize the c.e. reals $\beta$ such that $\mathcal{L}(\alpha) \cong \mathcal{L}(\beta)$ for a given $\alpha$.

\section{References}

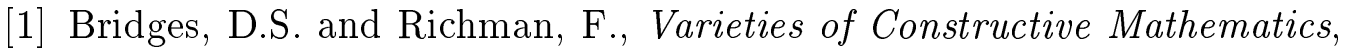
Cambridge University Press $\Gamma$ Cambridge $\Gamma 1987$.

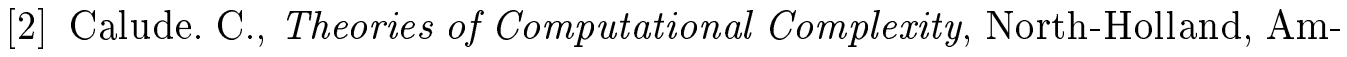
sterdam 1988 .

[3] C. S. Calude $\Gamma$ P. Hertling $\Gamma$ Computable approximations of reals: An information-theoretic analysis $\Gamma$ Fundamenta Informaticae, 33 (1998)Г116.

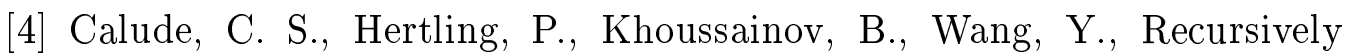
enumerable reals and Chaitin $\Omega$ numbers $\Gamma$ in $\mathrm{M}$. Morvan $\Gamma$ C. Meinel $\Gamma D$. Krob (eds.). STACS'98, Proceedings of the 15th Annual Symposium on Theoretical Aspects of Computer Science, Paris,1998ГLectures Notes in Computer Science 1373ГSpringer-VerlagГBerlinГ1998Г596-606.

[5] DowneyГR. and StobГМ.ГSplitting theorems in recursion theoryГAnnals of Pure and Applied Logic, 65 (1993). 1-106.

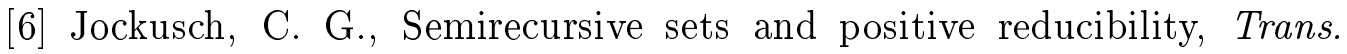
Amer. Math. Soc. 131 (1968)Г420-436.

[7] LermanГM. and RemmelГ J. В.ГThe universal splitting propertyГІГin

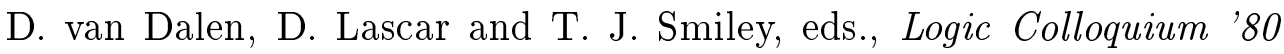

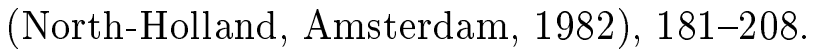

[8] LermanГM. and RemmelГJ. В.ГThe universal splitting propertyГІІГ J. Symbolic Logic 49 (1984)Г137-150.

[9] Martin-LöfTP.ГNotes on Constructive Mathematics $\Gamma$ Almqvist \& Wiksell Stockholm $\Gamma 1970$.

[10] Pour-ElГ M. B. and RichardsГ J. I.Г Computability in Analysis and Physics, Springer-Verlag $\Gamma$ Berlin $\Gamma 1989$.

[11] RiceГH. G.ГRecursive real numbers Г Proc. Amer. Math. Soc. 5 (1954)Г 784-791.

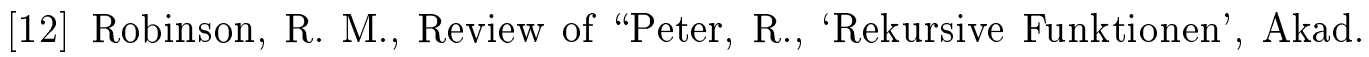

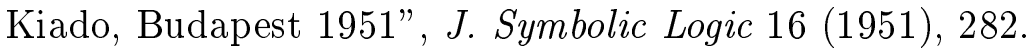




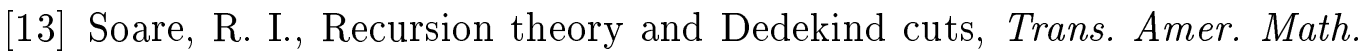
Soc. 140 (1969)Г271-294.

[14] SoareГR. I.ГRecursively Enumerable Sets and Degrees, Springer-Verlag $\Gamma$ New York $\Gamma 1987$.

[15] SpeckerГE.ГNicht konstruktiv beweisbare Sätze der AnalysisГJ. Symbolic Logic, 14 (1949)Г145-158.

[16] Turing $\Gamma$ A. M.Г On computable numbers $\Gamma$ with an application to the

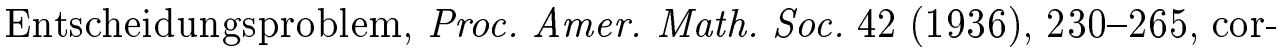
rections ibid. 43 (1937) 544-546.

[17] WeihrauchГК.ГComputability, Springer- VerlagГBerlinГ1987. 